\title{
Surface Photovoltage Effects in Photoemission from Metal-GaP(110) Interfaces: Importance for Band-Bending Evaluation
}

\author{
M. Alonso, R. Cimino, ${ }^{(a)}$ and K. Horn \\ Fritz-Haber-Institut der Max-Planck-Gesellschaft, D-1000 Berlin 33, West Germany
}

(Received 29 November 1989)

\begin{abstract}
Photoelectron spectra from metal overlayers on $\mathrm{GaP}(110)$ show that the photoionization light source may induce a surface photovoltage, causing an energy shift of valence- and core-level peaks. We analyze the dependence of this surface photovoltage on metal coverage, substrate doping, and temperature. The presence of a surface photovoltage seriously affects the determination of surface band bending by photoelectron spectroscopy, a technique which is generally thought to reflect the equilibrium electronic structure of metal-semiconductor interfaces.
\end{abstract}

PACS numbers: $79.60 . E q, 72.40 .+w, 73.30 .+y$

Despite a large number of experimental studies, the interpretation of Schottky-barrier (SB) heights at metalsemiconductor interfaces, and the evolution of surface band bending in the low-metal-coverage regime, remains a controversial issue. While most SB-height data for applications-oriented metal-semiconductor interfaces are obtained from $C / V$ or $I / V$ experiments, information about the electronic structure of the interface, as well as the evolution of the SB and its final height, is usually obtained through photoemission experiments. These are carried out on well-defined substrate surfaces and metal overlayers prepared in situ. ${ }^{1,2}$ In these studies, the surface band bending as a function of metal coverage, substrate doping, and temperature is derived from shifts in a substrate core-level line which may be detected even for thick overlayers (up to tens of $\AA$ ). Such shifts are interpreted as reflecting the position of the Fermi level in the band gap, with the implicit assumption that the condition of Fermi-level equilibrium is met. The possibility that the photoemission data could be affected by photon-induced electron-hole-pair creation and transport processes leading to a nonequilibrium charge distribution has been largely overlooked in these studies, in spite of observations that such effects may occur on clean as well as metal-covered semiconductor surfaces. ${ }^{3,4}$ Here we show how such processes strongly affect the thicknessdependent determination of surface band bending, and the measurement of SB heights by photoelectron spectroscopy, for metals deposited on $\mathrm{GaP}(110)$. This observation may have wider implications for the study of other metal-semiconductor interfaces.

All data were recorded with commercial angleresolving photoelectron spectrometers (ARIES by VSW Ltd., and ADES 400 by VG Scientific, Great Britain) on the TGM (toroidal grating monochromator) 5 and TGM 6 on the wiggler-undulator beam line as well as the 1-m Seya-Namioka monochromator, at the BESSY (Berliner Speicherring-Gesellschaft für Synchrotronstahlung) storage ring in Berlin. ${ }^{5}$ Overall resolution (electrons plus photons) on TGM 5 and 6 was about 60 $\mathrm{meV}$ at a photon energy of $55 \mathrm{eV}$, with photon flux about
$(1-7) \times 10^{13} \mathrm{~cm}^{-2} \mathrm{sec}^{-1}$ as evaluated from the yield of a gold photodiode; the flux on the 1-m Seya was approximately 2 orders of magnitude lower. The kinetic-energy scale of the spectra was calibrated taking into account the work function of clean polycrystalline gold; ${ }^{6}$ the photon energy was determined to within $0.05 \mathrm{eV}$ from corelevel emission excited by second-order light from the diffraction grating. If not otherwise specified, spectra were recorded under $50^{\circ}$ angle of incidence, and normal electron emission. Prenotched bars of GaP [n-(sulphur)-doped, carrier concentration of typically $2 \times 10^{17}-2 \times 10^{18} \mathrm{~cm}^{-3}$, and $p$-(zinc)-doped, carrier concentration of $(1.7-5.8) \times 10^{17} \mathrm{~cm}^{-3}$ ] were cleaved in situ, at an operating pressure of $5 \times 10^{-11}$ mbar. The crystals were mounted on a speciment holder which allowed cooling to about $100 \mathrm{~K}$ using liquid nitrogen. Two crystals, doped $n$ and $p$ type, were mounted close to each other, and were simultaneously exposed to the metal flux, thus ensuring similar amounts of metal deposition, and the exclusion of extraneous shifts due to differences in photon energy, etc. Metals were deposited from tungsten coils ( $\mathrm{Ag}, \mathrm{In})$ or a Knudsen cell (Al) at operating pressures of $1 \times 10^{-10} \mathrm{mbar}$ and below. Nominal thicknesses were measured by a quartz microbalance. The reference level for the photoelectron spectra (the Fermi energy of the electron analyzer) was measured by recording the valence-band spectrum of a gold foil in contact with the sample, which was cleaned in situ by scraping with a stainless-steel blade.

The fact that photon-induced nonequilibrium processes affect the determination of the surface band bending for $\mathrm{Ag} / \mathrm{GaP}(110)$ is evident from the core and valence spectra shown in Fig. 1. These data were recorded for a metal deposition equivalent to $5-\AA$ metal at room temperature for both $p$-type (upper curve) and $n$-type (lower curve) material. The corresponding spectra for the Fermi edge are shown for both doping types on the righthand side. We observe in Fig. 1 the unusual occurrence of photoelectrons at a kinetic energy above the reference Fermi level for $p$-type substrates (shifted by $\Delta_{p}$ ), while on the $n$-type substrates the Fermi-level emission occurs 


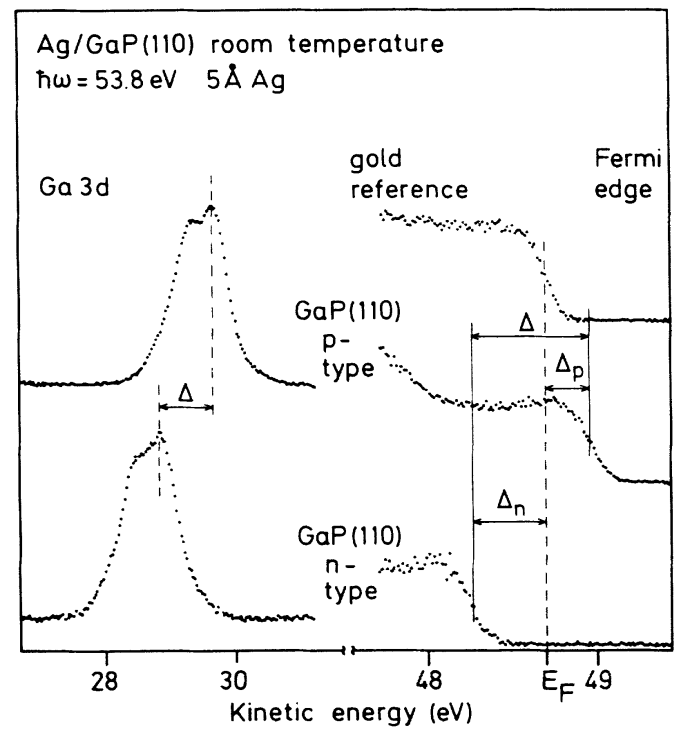

FIG. 1. Photoelectron spectra of a $6-\AA$-thick Ag film on $n$ and $p$-type $\mathrm{GaP}(110)$ in the region of the $\mathrm{Ga} 3 d$ level (lefthand side) and the valence-band maximum (right-hand side). Also shown is the spectrum from the Fermi edge of the gold foil in electrical contact with the samples.

below this level (shifted by $\Delta_{n}$ ). The total distance $\Delta=\Delta_{p}+\Delta_{n}$ is found to coincide to within $100 \mathrm{meV}$ with the distance between the $\mathrm{Ga} 3 d$ core-level lines (as shown in the figure). This indicates the existence of a shift different in sign for the two doping types and equally affecting emission features from both overlayer and substrate. The crystals were of sufficiently low resistivity (about $0.2 \Omega \mathrm{cm}$ ), and were carefully contacted by evaporating thin layers of $\mathrm{Au}-\mathrm{Ni}-\mathrm{Ge}$ alloy on the back of the samples. Thus charging can be excluded as a source for the shifts, as checked in experiments on the clean surface; it is also incompatible with the observed shifts because of the opposite sign of $\Delta_{n}$ and $\Delta_{p}$. This fact also rules out charging of the metal clusters such as observed for Ag on graphite by Wertheim, DiCenzo, and Youngquist. ${ }^{7}$ Similar results were obtained for In and $\mathrm{Al}$ overlayers on $\mathrm{GaP}(110)$, showing that the phenomenon is not limited to a particular choice of metal. The shifts $\Delta_{n}$ and $\Delta_{p}$ demonstrate that in the surface layer, a nonequilibrium situation is present. We contend that the shifts are due to the presence of a surface photovoltage (SPV), ${ }^{8,9}$ caused by the incident light used for photoexcitation.

A simplified picture of the influence of an SPV on the energies of valence and core levels as derived from photoemission experiments is visualized in Fig. 2 for $n$-type material. In an equilibrium situation, the presence of the metallic overlayer is assumed to give rise to a surface band bending $V_{b b}$ (left-hand side). ${ }^{1,2}$ The creation of electron-hole pairs by the incident light and/or secondary processes will lead to a nonequilibrium situation if recombination can be neglected; this is shown on the right-hand side of Fig. 2. The potential difference $V_{\mathrm{bb}}$

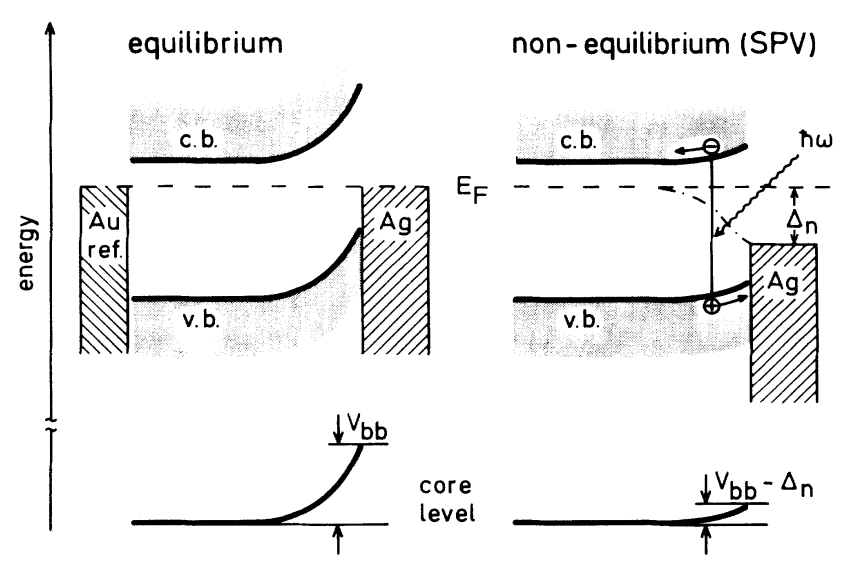

FIG. 2. Schematic drawing of the equilibrium level alignment (left-hand side), and the influence of the surface photovoltage on the energies of the substrate and overlayer peaks (right-hand side).

causes the electrons to be driven into the bulk whereas the holes are trapped near the surface, compensating the space charge, and producing a rigid shift of all semiconductor and metal levels; this situation can be described by a quasi Fermi level (dash-dotted line). ${ }^{10}$ It thus accounts for the occurrence of the overlayer metal Fermi edge at lower (or, for $p$-type material, at higher) energy than the gold reference Fermi level. This local charge compensation reduces the band bending caused by the metal overlayer. This is a self-limiting process since the surface band bending is needed to separate or trap the mobile carriers. ${ }^{3}$ The influence of this phenomenon, which is the physical basis of surface photovoltage spectroscopy $8,9,11$ on photoemission spectra has been described by Demuth et al. ${ }^{3}$ Previous investigations have shown that its magnitude depends on photon flux, surface recombination rate, temperature, and band bending. For a constant temperature, SPV increases exponentially with the magnitude of the fundamental gap. ${ }^{8,10}$ For clean silicon surfaces, Demuth et al. ${ }^{3}$ were able to completely compensate the band bending solely by irradiation with the uv source used for photoionization, by lowering the substrate temperature (to below $50 \mathrm{~K}$ ), which drastically reduces carrier recombination. This suggests that, while recombination has to be suppressed by lowering the temperature in clean silicon ${ }^{3}\left(E_{g}=1.12\right.$ $\mathrm{eV}$ ) in order to observe the SPV effect, the phenomenon can be observed at room temperature in $\mathrm{Ag}$-covered $\mathrm{GaP}(110)\left(E_{g}=2.25 \mathrm{eV}\right)$ as shown by the data in Fig. 1 . For metal-covered semiconductors, the study of $\mathrm{Ag}$ on $n$-type CdTe $(100)$ by John et al. ${ }^{4}$ reports a shift of the Fermi level in the photoelectron spectrum. Emission from the Fermi edge of $\mathrm{Ag}$ was observed at higher kinetic energies than that from a gold reference foil in contact with the sample. Note that while the SPV-induced shifts presented here show the directions expected on the basis of band bending induced by a metal, the trend observed 
for $\mathrm{Ag} / \mathrm{CdTe}(100)$ (Ref. 4) is contrary to this expectation, a point not elaborated upon by John et al. Hecht ${ }^{12}$ has estimated that there is a strong dependence of the magnitude of SPV on temperature, which in the case of GaAs leads to SPV saturation even at very low photon flux when the sample is cooled to below $200 \mathrm{~K}$.

Further evidence for the presence of a SPV is derived from an analysis of the dependence of $\Delta_{n}$ and $\Delta_{p}$ on important experimental parameters. An increase of overlayer thickness will lead to a reduction of photon flux and a concomitant decrease in SPV. This is in fact observed in the spectra of Fig. 3, for increasing Ag depositions as indicated. The clean surface shows the signature of the valence-band maximum (VBM). Deposition of $1.4 \AA$ leads to considerable emission above the valenceband maximum. At $4.3 \AA \mathrm{Ag}$, Fermi-edge emission occurs, shifted with respect to the gold reference by $\Delta_{n}$. For even higher depositions, this Fermi-edge emission progressively shifts towards the gold reference $E_{F}$, finally coinciding with it at a dose of $110 \AA \mathrm{Ag}$. That the gradual decrease of $\Delta_{n}$ is caused by the attentuation of the light intensity by the metallic overlayer is supported by experiments in which spectra were recorded at different angles of incidence of the light. Here, an increase in the magnitude of SPV was observed in going towards normal incidence, where the light penetrates much farther into the substrate. Lowering the substrate temperature leads to a pronounced increase of the magnitude of SPV. This is shown on the right-hand side of Fig. 3 for $n$ - as well as $p$-type substrates. The maximum shift $\Delta_{n}$ observed at low temperature (LT) is much larger than at room temperature (RT). A direct comparison of both temperature regimes is hindered by the different growth morphology at low temperatures, since the well-known tendency of the noble metals for clustering is suppressed at low temperature. Thus at depositions above $10 \AA$, patches of bare $\mathrm{GaP}(110)$ are still expected at room temperature, while the metal overlayer very likely extends over the entire semiconductor surface at $100 \mathrm{~K}$. This explains why the progressive shift of the Fermi edge towards the gold reference value is observed at much lower nominal coverages for LT than for RT. A detailed examination of the temperature dependence of SPV, which is beyond the scope of this Letter, will be reported elsewhere. $^{13}$

There is also a pronounced dependence of the magnitude of SPV on photon intensity at room temperature. For the preparation conditions shown in Fig. 1, a reduction of light intensity by a factor of 20 caused a decrease of $\Delta_{n}$ and $\Delta_{p}$ by about $0.1 \mathrm{eV}$, with a parallel shift of both Fermi-edge and core-level emission. In order to analyze the photon-flux dependence of SPV, similar measurements were also carried out on the Seya-Namioka monochromator using a photon flux of $10^{10}-10^{11}$ $\mathrm{cm}^{-2} \mathrm{sec}^{-1}$, well within the range of fluxes typically available in photoemission using synchrotron radiation.

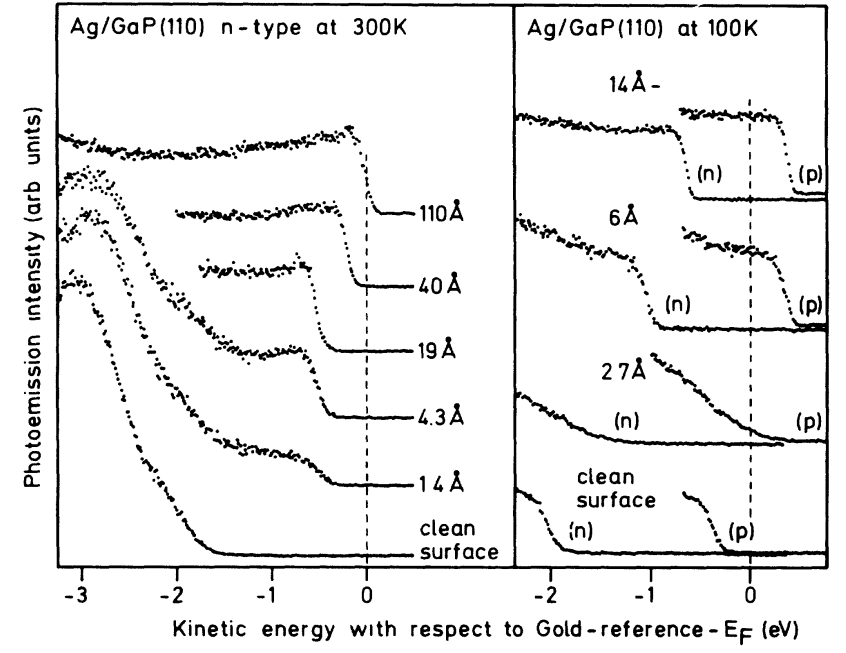

FIG. 3. Spectra of the valence-band edge of GaP(110) with different depositions of $\mathrm{Ag}$, at room temperature (left-hand side) and low (100 K) temperature (right-hand side), showing the magnitude of the SPV-induced shift as a function of $\mathrm{Ag}$ overlayer thickness.

Here, the maximum $\Delta_{n}$ for $\mathrm{Ag} / \mathrm{GaP}(110)$ at $\mathrm{RT}$ was $0.25 \mathrm{eV}$ compared with more than $0.5 \mathrm{eV}$ at the higher flux. While it is plausible that SPV should be reduced with decreasing photon flux, Hecht has estimated for $\mathrm{GaAs}$ that at $100 \mathrm{~K}$ a reduction of photon flux by 4 orders of magnitude only results in a decrease of SPV by less than $100 \mathrm{mV}$. This means that even at very low photon flux SPV effects in photoemission will still be appreciable at low temperature.

The large SPV observed here in photoelectron spectroscopy from Ag, In, and Al overlayers on $\mathrm{GaP}$ (110), and its persistence up to very high metal depositions ( $>40 \AA$ ) in particular, ${ }^{12}$ has important consequences for the determination of temperature-dependent surface band bending and Schottky-barrier-height determination by photoelectron spectroscopy. From the data such as shown in Fig. 3, combined with Ga $3 d$ spectra, we can obtain the energy for the surface Fermi level in the gap, corrected for the SPV-induced shift. ${ }^{4}$ This is shown as a function of metal deposition in Fig. 4 for $n$ - and $p$-type substrates at room temperature. Open circles (triangles) are for $n(p)$ substrates uncorrected for SPV, while solid symbols give the corrected values (corrections shown by arrows). Note that photoemission will not yield reasonable values about band bending at coverages when a Fermi edge has not been established, such that the correction $\Delta_{n}$ and $\Delta_{p}$ cannot be evaluated. The uncorrected values show the large difference between Fermi-level position for $n$ - and $p$-type substrates over a wide range of depositions. Correction by the SPV yields quite a different picture: The Fermi-level positions are quite close together even at low depositions, and are within less than $100 \mathrm{meV}$ once the sharp Fermi edge has evolved. It 


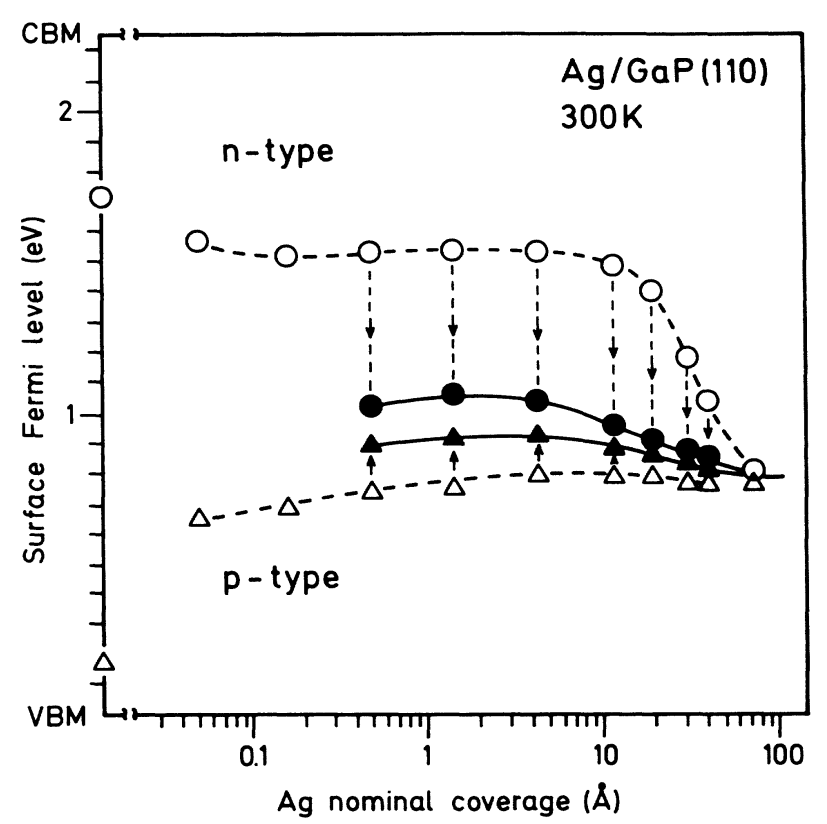

FIG. 4. Surface band bending and Schottky-barrier evolution in $\mathrm{Ag} / \mathrm{GaP}(110)$ at room temperature, as a function of $\mathrm{Ag}$ deposition. The open symbols show the uncorrected Fermilevel positions in the gap, while the solid symbols give the values corrected by the magnitude of the surface photovoltage.

is also important to note that the final Fermi-level position is only established at very high depositions, where substrate emission is barely detectable (minimum layer thickness more than $15 \AA$ ). Schottky-barrier-height determination is not affected as long as $\Phi_{b}$ is evaluated for very high metal coverages where SPV can be neglected. However, if one were to base the determination of the Schottky-barrier height $\Phi_{b}$ on coverages as low as 10 or $20 \AA,{ }^{14}$ the SPV would still strongly influence the determination of $\Phi_{b}$. This may be one reason why the Schottky barrier for $\mathrm{Ag}$ on $n$-type $\mathrm{GaP}(110)$ derived here $\left(\Phi_{b}=1.5 \mathrm{eV}\right)$ differs appreciably from that reported by Chiaradia et al. $\left(\Phi_{b}=1.08 \mathrm{eV}\right) .{ }^{14}$ A shift of Fermilevel emission relative to that of the external reference level has also been observed for different metals on $p$ type $\operatorname{InP}(110)$ at low temperature ${ }^{15}$ and $\mathrm{GaAs}(110)$ at low temperature. ${ }^{16}$ Thus it appears that the observation of SPV-induced shifts in photoemission may have wider and more general implications for the study of metal overlayers on semiconductors by this technique. It thus appears that the underlying assumption in previous photoemission work on band bending and Schottky-barrierheight determination, that the photoelectron spectra reflect the equilibrium valence-band arrangement, needs experimental confirmation in each case.

This work was supported by Bundesministerium für Forschung und Technologie under Grant No. 05241 HR as well as the Deutsche Forschungsgemeinschaft through Sonderforschungsbereich No. 6 project A 05. M.A. would like to thank the Consejo Superior de Investigaciones Cientificas for a fellowship. We would like to thank Ch. Maierhofer for help with some of the measurements, M. Prietsch and H. Lüth for interesting discussions, and $\mathrm{H}$. Haak for his technical assistance.

\footnotetext{
(a) Present address: Istituto di Struttura della MateriaConsiglio Nazionale delle Ricerche, Via E. Fermi, I-00044 Frascati, Italy.

'E. H. Rhoderick and R. Williams, Metal-Semiconductor Contacts (Clarendon, Oxford, 1988), 2nd ed.

${ }^{2}$ L. J. Brillson and G. Margaritondo, in The Chemical Physics of Solid Surfaces and Heterogenous Catalysis, edited by D. A. King and D. P. Woodruff (Elsevier, Amsterdam, 1988), Vol. 5; W. Mönch, J. Vac. Sci. Technol. B 6, 1270 (1988).

${ }^{3}$ J. E. Demuth, W. J. Thompson, N. J. DiNardo, and R. Imbihl, Phys. Rev. Lett. 56, 1408 (1986).

${ }^{4}$ P. John, T. Miller, T. C. Hsieh, A. P. Shapiro, A. L. Wachs, and T.-C. Chiang, Phys. Rev. B 34, 6704 (1986).

${ }^{5}$ W. Peatman, C. Carbone, W. Gudat, W. Heinen, P. Kuske, J. Pflüger, F. Schäfers, and T. Schroeter, Rev. Sci. Instrum. 60, 1445 (1989).

${ }^{6}$ D. E. Eastman, Phys. Rev. B 2, 1 (1970).

${ }^{7}$ G. K. Wertheim, S. B. DiCenzo, and S. E. Youngquist, Phys. Rev. Lett. 51, 2310 (1983).

${ }^{8}$ H. C. Gatos and J. Lagowski, J. Vac. Sci. Technol. 10, 130 (1973)

${ }^{9} \mathrm{G}$. Heiland and H. Lüth, Nuovo Cimento 39B, 748 (1977).

${ }^{10}$ S. M. Sze, Physics of Semiconductor Devices (Wiley, New York, 1982), 2nd ed.

${ }^{11}$ W. Mönch, P. Koke, and S. Krueger, J. Vac. Sci. Technol. 19, 331 (1981).

${ }^{12}$ M. Hecht, Phys. Rev. B 41, 7819 (1990).

${ }^{13} \mathrm{M}$. Alonso, R. Cimino, and K. Horn (unpublished).

${ }^{14}$ P. Chiaradia, M. Fanfoni, P. Nataletti, P. de Padova, R. E. Viturro, and L. J. Brillson, J. Vac. Sci. Technol. B 7, 195 (1989).

${ }^{15}$ C. Stephens and I. T. McGovern (private communication).

${ }^{16}$ R. Cimino, M. Alonso, and K. Horn (unpublished).
} 


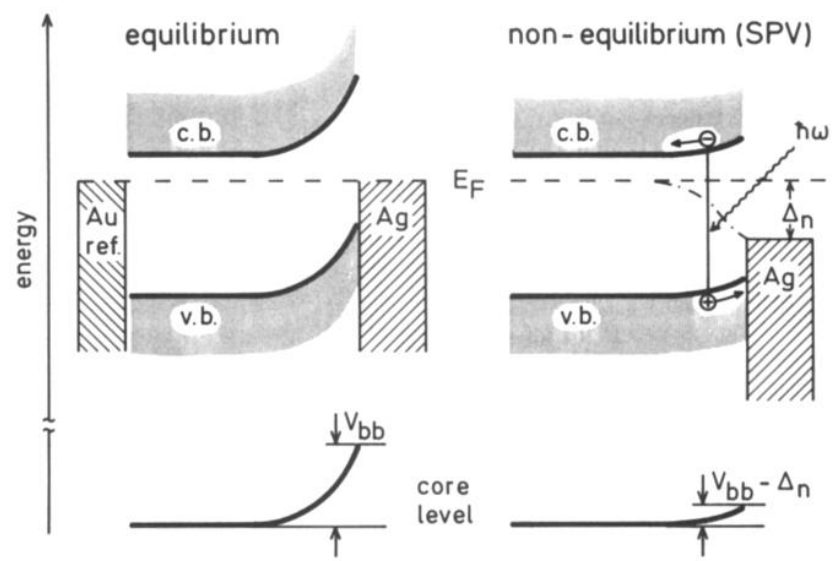

FIG. 2. Schematic drawing of the equilibrium level alignment (left-hand side), and the influence of the surface photovoltage on the energies of the substrate and overlayer peaks (right-hand side). 